${ }^{3}$ Radboud University Medical Centre, Nijmegen, Netherlands; ${ }^{4}$ Bicocca University, Milan, Italy; ${ }^{I}$ RCCS San Raffaele Scientific Institute, Milan, Italy

Background: Histiocytoses are disorders characterized by tissue infiltration by macrophages, dendritic cells, or monocyte-derived cells. These diseases are classified in five groups based on histologic, clinical, and molecular features: Langerhans-related, cutaneous/mucocutaneous and malignant histiocytoses, Rosai-Dorfman disease, and hemophagocytic lymphohistiocytosis (1). Langerhans-related histiocytoses comprise Langerhans cell histiocytosis and Erdheim-Chester disease, both inflammatory myeloid-driven diseases characterized by clonal activating mutations along the MAPK or related pathways, most commonly BRAFV600E, and by severe tissue and systemic inflammation (2). Here, we describe and characterize a novel, related histiocytosis chiefly manifested with severe synovial involvement.

Objectives: Here, we describe a novel histiocytosis whose histologic and clinical picture (severe synovial involvement, systemic inflammation, skin lesions, and diabetes insipidus) differed from known histiocytic disorder. In addition, we performed molecular studies aimed at identifying causative activating mutations. Finally, by means of a dynamic 3D tissue culture system we characterized immune-metabolic mechanisms underlying disease pathogenesis and clinical response to treatment.

Methods: The mutational status of oncogenes was determined with a mass spectrometry multiplexed genotyping approach (PentaPanel). Biospy samples were cultured in RCCSTM bioreactor (Synthecon) in the presence/absence of a MEK inhibitor (GSK1120212, 1nM), and then either processed for immunohistochemical analyses, or lysed for western blot analysis. Culture supernatants were collected for cytokine, chemokine and metabolome determination. The Bio-Plex Multiple-Cytokine Assay (Bio-Rad) and the Ella assay (ProteinSimple) were used to determine cytokine concentrations in supernatants and serum, respectively. Metabolomic studies were performed as described (3).

Results: We identified a causative mutation in the proto-oncogene KRAS (KRASG12D, not previously reported in related histiocytoses). In addition, 3D culture studies of patient's biopsies revealed KRAS-driven signaling, phenotypic, and immunometabolic features. These included constitutive ERK and AKT phosphorylation, up-regulated glucose metabolism with glycolysis and TCA cycle activation, and deregulated release of pro-inflammatory cytokines IL-1 $\beta$, IL- 6 and TNFa. All these features reverted upon pharmacologic inhibition of the MAPK pathway. Characterization of this novel condition instructed effective treatment of the patient with the MEK inhibitor cobimetinib.

Conclusion: Genetic, clinical, and histopathology features differentiate this condition from known histiocytic disorders. Mechanistically, KRASG12D causes constitutive activation of the MAPK pathway in macrophages, which results in maladaptive changes in cell energy metabolism sustaining rampant production of pro-inflammatory cytokines. Besides instructing effective treatment of this patient, these studies revealed metabolic rewiring as key to pathologic inflammatory activation of macrophages in human disease.

References:

[1] Emile JF, et al. Revised classification of histiocytoses. Blood. 2016

[2] Cavalli G, et al. The multifaceted clinical presentations and manifestations of Erdheim-Chester disease. Ann Rheum Dis. 2013

[3] Cavalli G, et al. Interleukin 37 reverses the metabolic cost of inflammation, increases oxidative respiration, and improves exercise tolerance. Proc Natl Acad Sci U S A. 2017

Disclosure of Interests: Giulio Cavalli Consultant of: SOBI, Pfizer, Sanofi, Novartis, Paid instructor for: SOBI, Novartis, Speakers bureau: SOBI, Novartis, Giacomo De Luca Speakers bureau: SOBI, Novartis, Celgene, Pfizer, MSD, Riccardo Biavasco Employee of: Bluebird, Travis Nemkov: None declared, Angelo D’Alessandro: None declared, Rob Arts: None declared, Antonello Villa: None declared, Daniela Belloni: None declared, Greta Grassini: None declared, Giulia Cangi: None declared, Claudio Doglioni: None declared, Elisabetta Ferrero: None declared, Marina Ferrarini: None declared, Lorenzo Dagna: None declared

DOI: 10.1136/annrheumdis-2020-eular.3170

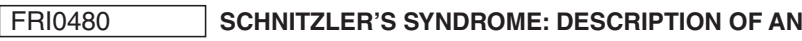 ITALIAN MULTICENTER COHORT}

F. Crisafulli ${ }^{1}$, P. Airò ${ }^{2}$, F. Franceschini ${ }^{1}$, A. Tincani ${ }^{1}$, M. Frassi ${ }^{2}$ on behalf of Working Group of Autoinflammatory Diseases of SIR (Italian Society of Rheumatology). ${ }^{1}$ ASST Spedali Civili and University of Brescia, Rheumatology and Clinical Immunology Unit, Brescia, Italy; ${ }^{2}$ ASST Spedali Civili of Brescia, Rheumatology and Clinical Immunology Unit, Brescia, Italy

Background: Schnitzler's syndrome is an autoinflammatory disease characterized by monoclonal gammopathy and recurrent episodes of urticaria accompanied by clinical and laboratory signs of acute inflammation. Although the exact pathogenic mechanisms have not been fully clarified, the role of Interleukin-1 seems to be central.

Objectives: To describe clinical features and therapeutic approach in patients with Schnitzler's Syndrome.

Methods: Retrospective analysis of an Italian multicenter cohort. Data are expressed as the median (IQR).

Results: The clinical data of 24 patients from 9 centers (median follow-up 6 years [2-10]; median age at diagnosis 56.5 years [51.25-64.25]) were collected. The median diagnostic delay was 2 years $(0-10)$; the diagnosis was made consensually at the onset of symptoms in 4 cases. The main clinical and laboratory features are shown in Table 1. Therapeutic response was evaluable in 20 patients: all received corticosteroids (CS; 25mg/day [25-50]); in one case, a good clinical response was observed. Eight patients were initially treated with colchicine: in 3 cases it was effective in controlling symptoms and reducing the dose of CS; other 8 patients were treated with csDMARDs ( $\mathrm{n}: 1$ [1-2]): only 1 patient had a good response to cyclosporin.

Table 1. Clinical and laboratory features

\begin{tabular}{lc}
\hline Chronic Urticarial Rash, n (\%) & $24 / 24(100)$ \\
\hline Pruritus, $\mathrm{n}(\%)$ & $17 / 24(71)$ \\
Intermittent fever, $\mathrm{n}(\%)$ & $23 / 24(96)$ \\
Arthralgia/Arthritis, n (\%) & $20 / 24(83)$ \\
Bone pain, $\mathrm{n}(\%)$ & $8 / 24(33)$ \\
Weight loss, $\mathrm{n}(\%)$ & $9 / 24(38)$ \\
Angioedema, n (\%) & $4 / 24(17)$ \\
Lymphoadenopathy, n (\%) & $7 / 24(29)$ \\
Hepatomegaly, n (\%) & $3 / 24(12)$ \\
Splenomegaly, n (\%) & $3 / 24(12)$ \\
Neuropathy, n (\%) & $4 / 24(17)$ \\
Raised ESR or CRP, n (\%) & $24 / 24(100)$ \\
Leukocytosis, n (\%) & $17 / 24(71)$ \\
Anemia, n (\%) & $9 / 24(38)$ \\
Monoclonal Gammopathy & \\
IgG $\lambda, \mathrm{n}(\%)$ & $5 / 22(23)$ \\
IgG $\kappa, \mathrm{n}(\%)$ & $6 / 22(27)$ \\
IgM $\lambda, \mathrm{n}(\%)$ & $1 / 22(5)$ \\
IgM $\kappa, \mathrm{n}(\%)$ & $12 / 22(55)$ \\
Bence Jones Protein, n (\%) & $6 / 23(26)$ \\
\hline
\end{tabular}

A bDMARD was initiated in 15 patients. In 7 of the 14 patients initially treated with anakinra this therapy was continued with benefit whereas in the othe 7 patients the treatment was discontinued for primary inefficacy (1 patient), secondary inefficacy ( 3 patients) and adverse events ( 3 patients; 2 injection site reaction, 1 severe allergic reaction). After anakinra discontinuation, 5 patients were treated with canakinumab with a good response in 3 cases and a partial response in 1 case (persistent arthritis); 1 patient died during the treatment. No response was observed in 3 patients treated with TNF inhibitors as a $2^{\text {nd }}$ or $3^{\text {rd }}$ line bDMARDs, as well as in 1 case initially treated with tocilizumab (in which a good response was afterwards obtained with canakinumab). bDMARDs were associated with a csDMARD in 2 patients (methotrexate and methotrexate + cyclosporine).

In one case monoclonal gammopathy evolved into Multiple Myeloma and the patient died 15 years after the onset of symptoms. Idiopathic myelofibrosis and myelodysplasia were found in one and in two patients, respectively.

Conclusion: In most cases csDMARDs and bDMARDs like anti-IL6 and antiTNFa were not able to control the disease. In contrast, in some cases, a good response to colchicine was observed; refractory patients may be successfully treated with anti-IL1 agents. Patients should be supervised for possible evolution towards lymphoproliferative disease.

Disclosure of Interests: None declared

DOI: 10.1136/annrheumdis-2020-eular.3770

\begin{tabular}{|l|l}
\hline FRI0481 & HEMOPHAGOCYTIC SECONDARY SYNDROME. \\
DIFFERENCES BETWEEN AUTOIMMUNE AND \\
HEMATO-ONCOLOGICAL ETIOLOGIES
\end{tabular}

C. A. Egües Dubuc ${ }^{1}$, A. De Diego $^{1}$, P. Cabrera Miranda ${ }^{2}$, N. Alcorta Lorenzo ${ }^{1}$, J. A. Valero Jaimes ${ }^{1}$, J. R. Furundarena Salsamendi ${ }^{3}$, L. M. Lopez Dominguez ${ }^{1}$, J. J. Cancio Fanlo ${ }^{1}$, O. Maiz-Alonso ${ }^{1}$, E. Uriarte Isacelaya ${ }^{1}$, J. Calvo ${ }^{4}$, J. M. Belzunegui Otano ${ }^{1} .{ }^{1}$ Donostia University Hospital, Rheumatology, Donostia, Spain; ${ }^{2}$ University Hospital October 12, Preventive Medicine and Public Health, Madrid, Spain; ${ }^{3}$ Donostia University Hospital, Haematology, San Sebastian, Spain; ${ }^{4}$ Araba University Hospital, Rheumatology, Vitoria, Spain

Background: The Hemophagocytic Syndrome (HPS) is has been classified into 2 groups: primary and secondary. The secondary form is mainly associated with hemato-oncological diseases (HOD) and autoimmune diseases (AID). The 
present work aims to obtain clinical and analytical data that can guide us to an etiological diagnosis.

Objectives: To describe and identify the differences between HPS secondary to AID and HOD during their admission to a tertiary hospital between 2005 and 2019.

Methods: This is a retrospective observational study. We include patient meeting the diagnostic criteria for HLH proposed by Henter Jl. (1), or who presented haemophagocytic cells $(\mathrm{HC})$ in the bone marrow biopsy (BMB), or who had HPS in the hospital discharge report. Demographic, clinical, analytical, etiological, underlying disorders and prognosis variables were collected. Continuous variables are described with the mean or median according to the degree of normality. Kruskal Wallis, Fisher test and Mann-Whitney $U$ test were used for the bivariate analysis, and also a multivariate logistic regression analysis was performed.

Results: We found 30 patients with secondary HPS, 22 of which corresponded to the AID [Systemic Lupus Erythematosus $(n=5)$, Adult Still's Disease $(n=3)$, Rheumatoid arthritis $(n=1)$ and IgG4 Sclerosing Disease $(n=1)$ ] and HOD [Non-Hodgkin's Lymphoma $(n=3)$, Myelodysplastic syndrome $(n=3)$, Acute leukemia $(n=3)$, Extranodal NK cell lymphoma $(n=1)$, Multiple Myeloma $(n=1)$ and probable lymphoproliferative process $(n=1)]$. The coincidence of an infectious disease with HPS was observed in 8 of the 22 cases [AID: 5 cases (2 Cytomegalovirus, 2 viral respiratory infections and 1 bacterial infection) and HOD: 3 cases (2 Epstein Barr virus and 1 bacterial infection)]. In two patients with HPS secondary to HOD (acute leukemia), allogeneic transplantation was associated as a possible trigger. In a patient with myelodysplastic syndrome, HPS was associated with the development of graft versus host disease. The age at diagnosis was lower in the AID [40 (26.5 - 56.3); p 0.001]. The HOD had more severe cytopenias [platelets 4500 (650 - 15,750; p 0.009), leukocytes 2050 (20 - 728; p 0.0001) and neutrophils $0(0-280 ; p$ 0.002)]. Overall mortality ( $n=30$ patients) was $43.3 \%$ (HOD: 66.7\%; p 0.029) (table 1). In the final multivariate model according to AID and HOD, the following independent associations were observed: age ( $p 0.002)$, platelets ( $p$ 0.031), GOT (p 0.012), GPT ( $p$ 0.015), total proteins ( $p$ 0.007) and mortality ( $p$ 0.007)

Conclusion: The HOD presented higher mortality and severe cytopenias. The AID presented a higher elevation of transaminases and better prognosis. References:

[1] Henter Jl, et al. HLH-2004: Diagnostic and therapeutic guidelines for HLH. Pediatr Blood Cancer. 2007;48:124.

Disclosure of Interests: César Antonio Egües Dubuc: None declared, Andrea De Diego: None declared, Patricia Cabrera Miranda: None declared, Nerea Alcorta Lorenzo: None declared, Jesús Alejandro Valero Jaimes: None declared, Jose Ramon Furundarena Salsamendi: None declared, Luis Maria Lopez Dominguez: None declared, Jorge Jesús Cancio Fanlo: None declared, Olga Maiz-Alonso: None declared, Esther Uriarte Isacelaya: None declared, Jaime Calvo Grant/research support from: Lilly, UCB, Consultant of: Abbvie, Jansen, Celgene, Joaquin Maria Belzunegui Otano: None declared

DOI: 10.1136/annrheumdis-2020-eular.4688

\section{FRI0482 \\ A SYSTEMATIC REVIEW FOR THE MANAGEMENT OF THE GENETICALLY DEFINED IL-1-MEDIATED AUTOINFLAMMATORY DISEASES, CAPS, TRAPS, MKD AND DIRA}

R. Berard ${ }^{1}$, M. Romano ${ }^{2}$, Z. S. Arici ${ }^{3}$, D. Piskin ${ }^{1}$, O. Jones ${ }^{4}$, K. Durrant ${ }^{5}$, R. Goldbach-Mansky ${ }^{6}$, M. Gattorno ${ }^{7}$, E. Demirkaya ${ }^{1}{ }^{1}{ }^{1}$ Western University, London, Canada; ${ }^{2}$ stituto Ortopedico Gaetano Pini, Milano, Italy; ${ }^{3}$ Sanliurfa Education and Research Hospital, Sanliurfa, Turkey; ${ }^{4}$ Walter Reed Army Medical Center, Washington, United States of America; ${ }^{5}$ Autoinflammatory Alliance, San Francisco, United States of America; ${ }^{6} \mathrm{NIH}$ Clinical Center, Bethesda, United States of America; ${ }^{7}$ Istituto Giannina Gaslini, Genova, Italy

Background: Ultra-rare genetically defined IL-1 mediated autoinflammatory diseases (AIDs) include mevalonate kinase deficiency (MKD), tumor necrosis factor receptor associated periodic syndrome (TRAPS), cryopyrinopathies (CAPS) and deficiency of the IL-1 receptor antagonist (DIRA). These disorders start perinatally, the clinical disease manifestations include systemic inflammation; and late diagnosis and inappropriate treatment cause irreversible organ damage. The varying skills of treating rheumatologists and paediatricians illustrate the need for management guidance, however criteria for validated methodology is geared towards common diseases with more heterogeneous pathogenesis.

Objectives: The focus of this systematic review includes the evaluation of the existing literature and the evaluation of existing EULAR methodology for use in the ultra-rare diseases with defined pathomechanisms, CAPS, TRAPS, MKD and DIRA

Methods: EULAR standardized operating procedures were followed during the review, including a meeting of experts to discuss key words, inclusion/exclusion criteria and PICO questions. Three fellows established the protocol of the review under the supervision of the EULAR methodologist and PubMed, Embase, and Cochrane databases were searched up to September 30, 2019.

Results: We found 1582 articles for CAPS, 1109 articles for TRAPS, 1741 articles for MKD and 557 articles for DIRA. Duplications, animal models and basic science studies, conference papers, systematic reviews/meta-analysis and articles not in English language is excluded. If we excluded case reports $(n<4)$, then 72 articles for CAPS, 40 articles for TRAPS, 44 articles for MKD and 1 article for DIRA should be included for full text evaluation and data extraction (Figure 1) However among the case reports, patients excluded achieved complete remission, assessed by clinical criteria and biomarkers. Of the studies included only few randomized studies for CAPS, TRAPS, MKD, and DIRA and would achieve higher level of evidence (Figure 1).

Conclusion: CAPS, TRAPS, MKD and DIRA are monogenic diseases with defined pathways and outcomes that include inflammatory remission based on clinical and biomarker data. Current methodological evaluations for genetically complex diseases undervalue the published evidence in case reports that report on remission and IL-1 biomarkers. We suggest that case studies that include hard outcomes including inflammatory remission, and open label withdrawal studies that are both backed by biomarkers could be allowed to be included and be considered for a stronger evidence level.

Table 1. Characteristics and comparative analysis of HPS secondary to AID and HOD

\begin{tabular}{|c|c|c|c|c|c|c|c|}
\hline \multirow[b]{2}{*}{$n$} & \multicolumn{2}{|c|}{ Total } & \multicolumn{2}{|c|}{ AID } & \multicolumn{2}{|c|}{ HOD } & \multirow[b]{2}{*}{${ }^{*} p<0,05$} \\
\hline & \multicolumn{2}{|c|}{30} & \multicolumn{2}{|c|}{10} & \multicolumn{2}{|c|}{12} & \\
\hline Age $(x \pm s)$ & 55,5 & $\pm 18,3$ & 40 & $26,5-56,3$ & 68 & $57,5-73,8$ & 0,001 \\
\hline Gender, male & 14 & $46,7 \%$ & 3 & $30 \%$ & 9 & $75 \%$ & 0,084 \\
\hline Spenomegaly & 16 & $53,3 \%$ & 5 & $50 \%$ & 8 & $66,7 \%$ & 0,666 \\
\hline Hepatomegaly & 10 & $33,3 \%$ & 4 & $40 \%$ & 4 & $33,3 \%$ & 1,000 \\
\hline $\mathrm{Hb}(\mathrm{g} / \mathrm{dL})$ & 7,1 & $6,4-7,9$ & 7,2 & $6,6-8,4$ & 6,5 & $5,9-7,3$ & 0,05 \\
\hline $\mathrm{Pt}\left(\mathrm{x} 10^{9} / \mathrm{L}\right)$ & 13500 & $\begin{array}{c}5000-52 \\
500\end{array}$ & 31650 & $\begin{array}{c}11000-100 \\
250\end{array}$ & 4500 & $650-15750$ & 0,004 \\
\hline Leu $\left(x 10^{9} / \mathrm{L}\right)$ & 1250 & $238-3153$ & 1985 & $1350-3382$ & 185 & $20-728$ & 0,000 \\
\hline $\mathrm{Neu}\left(\times 10^{9} / \mathrm{L}\right)$ & 615 & $0-1550$ & 948 & 633-1 808 & 0 & $0-280$ & 0,001 \\
\hline $\mathrm{Fb}(\mathrm{mg} / \mathrm{dL})(\mathrm{n}=24)$ & 171 & $111-358$ & 212 & $90-450$ & 167 & $114-354$ & 1,00 \\
\hline \multirow[t]{2}{*}{ Fer $(\mathrm{ng} / \mathrm{mL})(\mathrm{n}=28)$} & 15330 & $5434-38$ & 14263 & $4254-14263$ & 16796 & $8287-56$ & 0,314 \\
\hline & & 284 & & & & 969 & \\
\hline $\operatorname{Tg}(\mathrm{mmol} / \mathrm{L})$ & 341 & $226-438$ & 411,5 & $234-572$ & 321 & $233,8-403,8$ & 0,314 \\
\hline GOT (U/L) & 139 & $78-406$ & 457 & $289-1140$ & 106 & $71-193$ & 0,003 \\
\hline GPT (U/L) & 162 & $46-388$ & 432 & $174-599$ & 109 & $54-263$ & 0,017 \\
\hline T.P. $(n=29)$ & 4,8 & $\pm, 1,04$ & 5,0 & $4,5-5,8$ & 4,3 & $3,9-4,5$ & 0,003 \\
\hline Hospital stay & 35,5 & $20,0-60,8$ & 30,5 & $9,5-53,3$ & 61,5 & $29,3-93,3$ & 0,036 \\
\hline Hospital stay pre-dx & 16,5 & $8,5-29,8$ & 10 & $5,0-16,5$ & 26 & $10-39$ & 0,038 \\
\hline Mortality & 13 & $43,3 \%$ & 1 & $10 \%$ & 8 & $66,7 \%$ & 0,011 \\
\hline
\end{tabular}

Hb: hemoglobin, Pt: platelets, Leu; leukocytes, Neu, neutrophils, Fb: fibrinogen, Fer: ferritin, Tg: triglycerides, GOT: aspartate aminotransferase, GPT: alanine aminotransferase, T.P.: total proteins, pre-dx: prior to the diagnosis of HPS according to BMO. *Analysis between AID and HOD. 